\title{
NONNEGATIVE MATRICES WITH NONNEGATIVE INVERSES
}

\author{
RALPH DEMARR
}

\begin{abstract}
We generalize a result stating that a nonnegative finite square matrix has a nonnegative inverse if and only if it is the product of a permutation matrix by a diagonal matrix. We consider column-finite infinite matrices and give a simple proof using elementary ideas from the theory of partially ordered linear algebras.
\end{abstract}

In [1] the authors show that a nonnegative square matrix has a nonnegative inverse if and only if its entries are all zero except for a single positive entry in each row and column. In this note we generalize this result and simplify the proof as well.

Let $A$ denote the real linear algebra of all column-finite infinite matrices with real entries. We partially order $A$ as follows: $\left[\alpha_{i j}\right] \leqq\left[\beta_{i j}\right]$ if and only if $\alpha_{i j} \leqq \beta_{i j}$ for all $i, j$. Thus, $A$ is a partially ordered linear algebra (pola) and if 1 denotes the identity matrix, then $0 \leqq 1$. See [2] for the precise definition of a pola. An example will illustrate the result to be obtained. Let $x=\left[\alpha_{i j}\right]$ and $y=\left[\beta_{i j}\right]$ be defined as follows: $\alpha_{i j}=1$ if $i=j+1$ and is zero otherwise; $\beta_{i j}=1$ if $j=i+1$ and is zero otherwise. Thus, $0 \leqq x, 0 \leqq y$ and $0 \leqq x y \leqq 1 \leqq y x$. Note that each column of $x$ contains exactly one positive entry and each row of $x$ contains at most one positive entry.

THEOREM. Let $A$ be the pola described above. If $x, y \in A, 0 \leqq x, 0 \leqq y$ and $0 \leqq x y \leqq 1 \leqq y x$, then each column of $x$ contains exactly one positive entry and each row of $x$ contains at most one positive entry. The conclusion applies to the matrix $y$ if we interchange the words "row" and "column".

Proof. Define $d=y x-1 \geqq 0$ and note that $1+d \leqq(1+d)^{2}=y x y x \leqq y x=$ $1+d$ since $x y \leqq 1$. Hence, $1+2 d \leqq(1+d)^{2} \leqq 1+d$, which means $d \leqq 0$. Thus $d=0$ and $y^{\prime} x=1$, which means that $y$ is a left inverse for $x$. Hence, each column of $x$ must contain at least one positive entry. Next construct a matrix $z$ so that $0 \leqq z \leqq x$ and each column of $z$ has only one positive entry and this entry is equal to the corresponding entry in the matrix $x$.

Received by the editors October 1, 1971.

AMS 1970 subject classifications. Primary 15A09; Secondary 15A45, 15 A48.

Key' 'ords and phrases. Matrix theory, inverses, ordered algebras. 
Note that $0 \leqq z y \leqq x y \leqq 1$, which means that $z y$ and $x y$ are diagonal matrices. Hence, $(z y)(x y)=(x y)(z y)$. Now $z=(z y)(x y) x=(x y)(z y) x=x(y z)$ and $0 \leqq y z \leqq y x=1$, which means that $y z$ is a diagonal matrix. Using elementary properties of matrix multiplication and the fact that $x$ and $z$ have one positive entry in common in each column we see that $y z=1$ and therefore $x=z$. Hence, $x$ has exactly one positive entry in each column.

The example above shows that some rows of $x$ may contain only zeros. We show that $x$ has at most one positive entry in each row. Let us now construct a matrix $w$ so that $0 \leqq u \leqq x$ and each row of $w$ has only one positive entry if the same row of $x$ has a positive entry in it and this entry is equal to the corresponding entry in the matrix $x$. Now $w=$ (wy) $x$ and since $0 \leqq n y \leqq x y \leqq 1$, we see that $w y$ is a diagonal matrix. The same reasoning applied above to the matrix $z$ shows that $w=x$. Hence, $x$ has at most one positive entry in each row.

\section{REFERENCES}

1. I. A. Brown, M. Juncosa and V. Klee, Invertibly positive linear operators on spaces of continuous functions, Math. Ann. 183 (1969), 105-114. MR 42 \#8314.

2. R. E. DeMarr, Convergence of a sequence of powers, Proc. Amer. Math. Soc. 23 (1969), 401-403. MR 39 \#6805.

Department of Mathematics, University of New Mexico, Albuquerque, New MeXICO 87106 